\title{
Calculating the light by light contribution to the muon anomalous magnetic moment using lattice QED
}

\section{Saumitra Chowdhury*}

Physics Department, University of Connecticut, Storrs, CT 06269-3046, USA

E-mail: saumitra@phys.uconn.edu

\section{Thomas Blum}

Physics Department, University of Connecticut, Storrs, CT 06269-3046, USA

RIKEN-BNL Research Center, Brookhaven National Laboratory, Upton, New York 11973, USA

E-mail: tblumephys.uconn.edu

\section{Taku Izubuchi}

Kanazawa University, Institute for Theoretical Physics, Kanazawa 920-1192, Japan

RIKEN-BNL Research Center, Brookhaven National Laboratory, Upton, New York 11973, USA

E-mail: izubuchi@quark.phy.bnl.gov

\section{Masashi Hayakawa}

Department of Physics, Nagoya University, Nagoya 464-8602, Japan

E-mail: hayakawa@eken.phys.nagoya-u.ac.jp

\section{Norikazu Yamada}

High Energy Accelerator Research Organization (KEK), Tsukuba, Ibaraki 305-0801, Japan

The Graduate University for Advanced Studies, Tsukuba, Ibaraki 305-0801, Japan

E-mail: norikazu.yamada@kek.jp

\section{Takeshi Yamazaki}

Yukawa Institute for Theoretical Physics (YITP), Kyoto University, Kyoto 606-01, Japan

E-mail: tyamazak@yukawa.kyoto-u.ac.jp

We report on an attempt to calculate the light-by-light contribution to the muon anomalous magnetic moment in QED, using lattice techniques, as a first but significant step toward computing the analogous hadronic contribution. We use domain wall fermions on quenched non-compact QED configurations. The method is discussed in detail, and preliminary results are presented. A signal is not obtained with the limited statistics accumulated so far. We mention possible ways to improve the statistics to obtain a non-zero result.

The XXVI International Symposium on Lattice Field Theory

July 14 - 19, 2008

Williamsburg, Virginia, USA

\footnotetext{
${ }^{*}$ Speaker.
} 


\section{Background \& Motivation}

The magnetic dipole moment of muon is one of the most precisely measured and calculated quantities in elementary particle physics. It has been measured to a precision of 0.54 parts per million (ppm) at Brookhaven National Lab (BNL) [1]. The Standard Model prediction has reached a comparable level to the measured quantity. The difference between the theoretical and measured quantities can be attributed to possible new physics such as Super-Symmetry (SUSY).

The magnetic dipole moment of muon of mass $m_{\mu}$ and charge $e$ is given by $\vec{\mu}=g\left(\frac{e}{2 m_{\mu}}\right) \vec{S}$, where $g$ and $\vec{S}$ are the gyromagnetic ratio and muon spin respectively. Quantum Electro-Dynamics (QED) predicts $g=2$ at the tree level for an elementary spin- $\frac{1}{2}$-particle such as the muon. Quantum corrections from QED, from strong or weak interactions, or from possible new physics lead to a contribution of $a=(g-2) / 2$ which is called the anomalous magnetic dipole moment. The theoretical prediction of the one-loop QED contribution to $a$ of a lepton is given by the well-known Schwinger term, $a=\alpha / 2 \pi$. This dominant contribution is then also further subjected to higherorder QED and QCD (Quantum Chromo-Dynamics) corrections. The loop contributions from heavier particles with mass $M_{\text {NewPhys. }}$ are suppressed by $m_{\mu}^{2} / M_{\text {New Phys. }}^{2}$, where $M_{\text {NewPhys. }}$ is SUSY particle mass. Therefore, the anomalous magnetic dipole moment of muon is $\left(m_{\mu} / m_{e}\right)^{2} \approx 40000$ times more sensitive to new physics than that of electron.

The anomalous magnetic dipole moment has been measured in the $g-2$ experiment E821 with great accuracy at BNL [1]

$$
a_{\mu}(E X P)=11659208(6.3) \times 10^{-10} .
$$

The Standard Model prediction in [2], based on the $e^{+} e^{-} \rightarrow$ hadrons cross-section is

$$
a_{\mu}^{S M}=11659184.1(7.2)^{\text {Vac.Pol. }}(3.5)^{L B L}(0.3)^{\text {QED } / \text { Weak }} \times 10^{-10}
$$

where the superscripts correspond to errors due to hadronic vacuum polarization, hadronic light-bylight scattering, QED and weak interactions repectively. The difference between the SM prediction and the experimental result is

$$
\Delta(E X P-S M)=23.9(9.9) \times 10^{-10}
$$

which suggests the reduction of the uncertainty attributed to the hadronic contributions.

The hadronic light-by-light contribution is very difficult to evaluate. Current estimates vary between model calculations, $8.6(3.5) \times 10^{-10}$ [3] to $13.6(2.5) \times 10^{-10}$ [4]. Evaluating the lightby-light contribution with greater accuracy may help reduce the discrepancy and hint to underlying structures of possible new physics.

\section{Proposed method}

Our proposal, based on [5] and summarized here, is to evaluate the hadronic light-by-light scattering amplitude depicted in Fig. 1, which gives rise to an $\mathscr{O}\left(\alpha_{\mathrm{em}}^{3}\right)$ contribution to the muon $g-2$, where $\alpha_{\mathrm{em}} \equiv e^{2} /(4 \pi)$. 


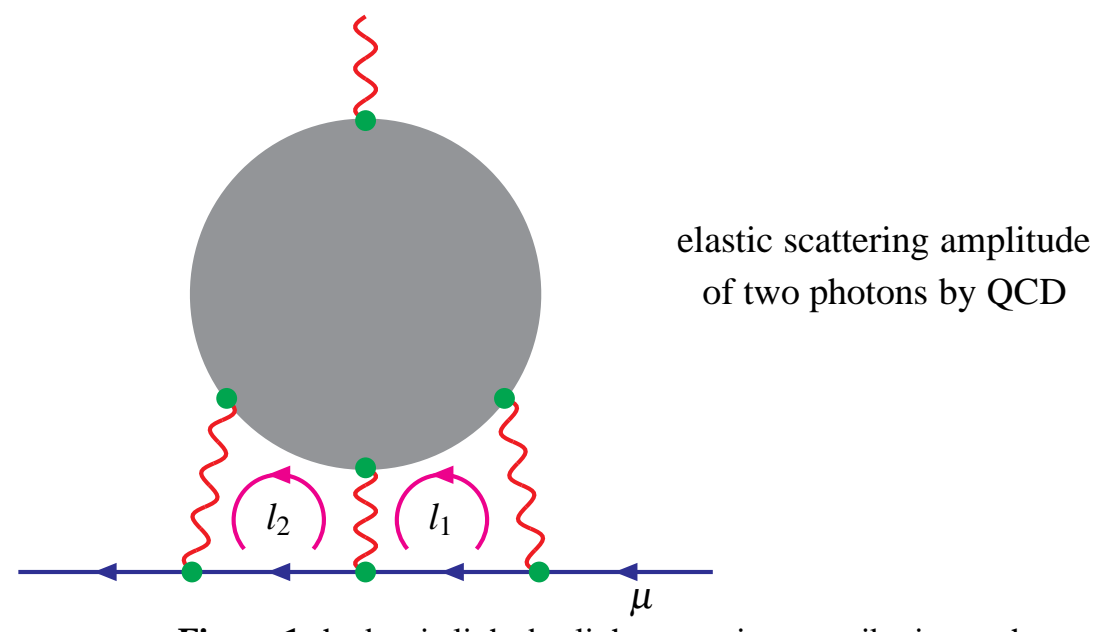

Figure 1: hadronic light-by-light scattering contribution to the muon $g-2$

The diagram can be computed using the following approach; the correlation of four electromagnetic currents are calculated repeatedly, using lattice QCD techniques, for two independent momenta $l_{1}, l_{2}$, of two of the three off-shell photons, and are then integrated over $l_{1}, l_{2}$ in a perturbative QED framework. This direct lattice calculation is numerically expensive since it requires spacetime volume-squared independent momentum-source calculations (This approach is discussed in [6].).

An alternative approach, the one we take in this work, is to use combined (QED+QCD) lattice simulation to calculate the entire diagram containing the external muon line in the non-perturbative framework. This approach can be written as

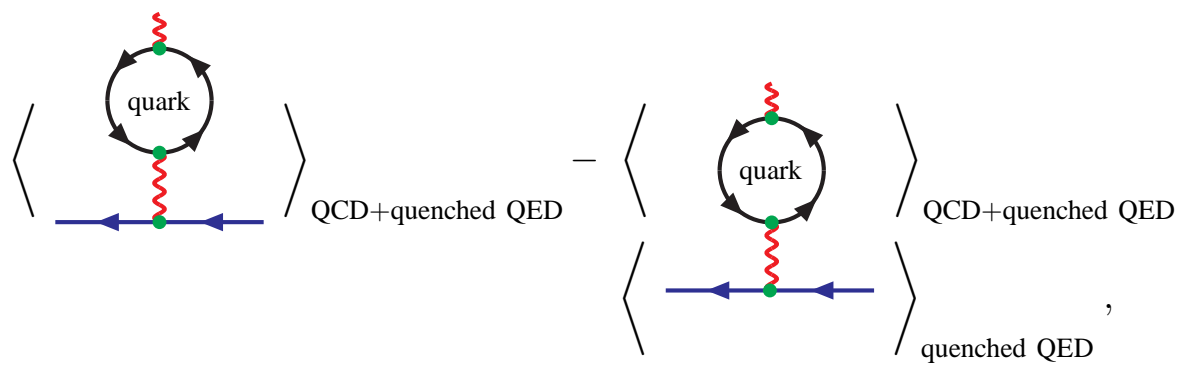

where the red, black, and blue lines represent the free photon, full quark, and muon propagators, respectively, with some proper gauge fixing condition. The average $\langle$,$\rangle means the one over the$ unquenched $S U(3)_{C}$ and/or the quenched $U(1)_{\text {em }}$ gauge configurations.

Let us look at the first term of Eq. (2.1) perturbatively with respect to QED in order to explain the underlying mechanism regarding the proposed method. The Feynmann diagrams contributing to the anomalous magnetic moment is depicted in Eq. (2.2). The left diagram in the first line gives the $\mathscr{O}\left(\alpha_{\mathrm{em}}^{2}\right)$-contribution. The $\mathscr{O}\left(\alpha_{\mathrm{em}}\right)$-corrections to its muon part and to its quark part induce $\mathscr{O}\left(\alpha_{\text {em }}^{3}\right)$-contributions shown in the right diagram on the first line as well as on the second line respectively. We recall that the QED gauge configurations in the first term of Eq. (2.1) are commonly shared by the quark part and the muon part. Hence, the photons can be exchanged between the two parts. As a consequence, the left diagram in the second line of Eq. (2.2) is induced at $\mathscr{O}\left(\alpha_{\mathrm{em}}^{3}\right)$, which takes the form of our target, Fig. 1. On the other hand, in the remaining two $\mathscr{O}\left(\alpha_{\mathrm{em}}^{3}\right)$ diagrams the quark and muon parts are connected only by a single photon line. Since 
these diagrams commonly contribute to both the first and second terms in Eq. (2.1), only the lightby-light diagram remains un-cancelled in the difference of Eq. (2.1). Thus, we may extract the light-by-light contribution.

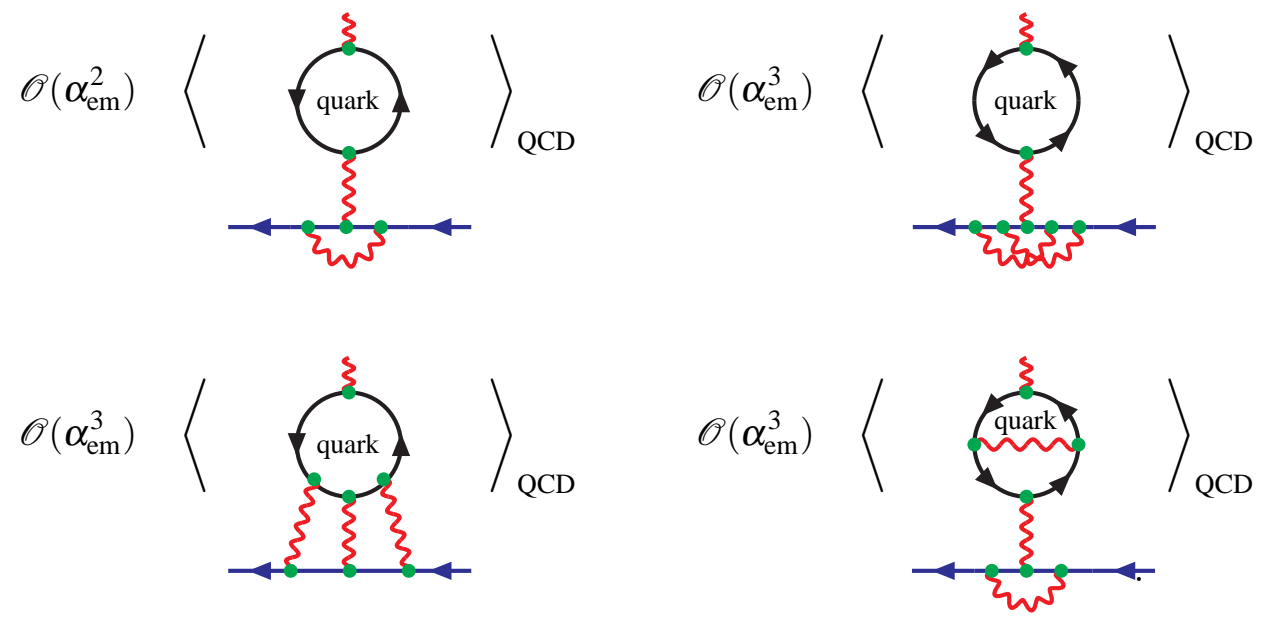

\section{Lattice Implementation}

In this section, we describe our computational approach, depicted in Fig. (2) for pure QED, i.e. the hadronic blob is replaced by a lepton loop for now. The reason behind this is to compare the lattice calculation with the well known QED result. Once the lattice QED calculation is understood and is in agreement with the perturbative result, we can simply switch back to the quark loop. The loop is created from a local domain wall fermion (DWF) point source, with conserved currents in the internal vertices of the lepton loop and line. The use of the conserved currents is crucial to avoid the appearance of quadratic divergences.

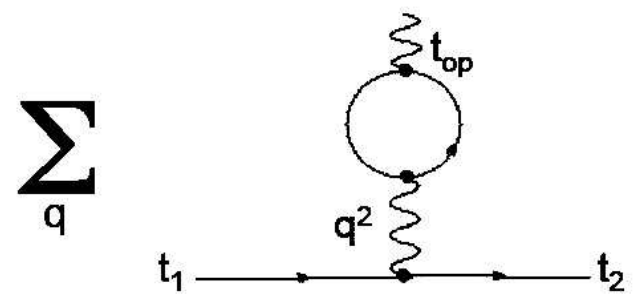

Figure 2: Lattice implementation of the light-by-light diagram.

The definitions of the local and point-split conserved currents are given by

$$
\begin{gathered}
j_{x, \mu}=\bar{q}_{x} \gamma_{\mu} q_{x} \\
J_{x, \mu}=\frac{1}{2} \sum_{s=0}^{L_{s}-1}\left(\bar{\Psi}_{x+\mu, s}\left(1+\gamma_{\mu}\right) U_{x, \mu}^{\dagger} \Psi_{x, s}-\bar{\Psi}_{x, s}\left(1-\gamma_{\mu}\right) U_{x, \mu} \Psi_{x+\mu, s}\right),
\end{gathered}
$$

where $q_{x}$ and $\Psi_{x, s}$ correspond to four and five dimensional fermionic fields, respectively, and the sum is taken over the sites of the fifth dimesion. The amplitude for the loop in configuration space is given by

$$
\mathscr{M}_{\text {loop }}=\Pi^{\mu v}(x, y)
$$


where $x$ and $y$ are the external and internal space-time points. The Fourier transformation at the internal vertex gives $\Pi^{\mu v}(x, q)$ where $q$ is the four momentum of the photon propagator joining the loop with the line. Because a point source is used at $x$, all possible momenta at that point are allowed.

The muonic line is constructed from momentum source propagators. The incoming propagator is created at time $t_{1}$ with $\vec{p}=0$, while the outgoing propagator with one unit of momentum is destroyed at a later time $t_{2}$. The two are joined at the vertex by the insertion of a conserved current. The amplitude for the line in configuration space is then given by

$$
\mathscr{M}_{\text {line }}=\Gamma^{\rho}\left(t_{1}, \overrightarrow{0} ; z ; t_{2}, \vec{p}\right)
$$

where $z$ is the location of the internal vertex. Again, a Fourier transformation at this vertex yields $\Gamma^{\rho}\left(t_{1}, \overrightarrow{0} ;-q ; t_{2}, \vec{p}\right)$. Multiplying the two amplitudes together, and by the photon propagator, summing over all momenta $q$, and averaging over all gauge fields yields

$$
\mathscr{M}=\left\langle\sum_{q \neq 0} \Pi^{\mu v}\left(t_{\mathrm{op}}, \vec{p}, q\right) \frac{g^{v \rho}}{q^{2}} \Gamma^{\rho}\left(t_{1}, \overrightarrow{0} ;-q ; t_{2}, \vec{p}\right)\right\rangle .
$$

The configuration average ensures spatial momentum conservation, and the external muons are put on-shell in the usual way by taking $t_{1} \ll t_{o p} \ll t_{2}$. The subtraction term is computed by averaging the loop and line separately, and then multiplying the averages together.

To check the validity of the code using conserved current, we check to see that the WardTakahashi Identities (WTI) are satisfied for both the muon loop and line on an arbitrary configuration. The loop must satisfy

$$
q^{v} \Pi^{\mu v}(x, q)=0
$$

where $q^{v}=2 \sin \left(\pi n_{v} / L\right)$ is the lattice momentum. The equation for the line is bit more complicated since the contact terms from the two sources do not cancel.

$$
\begin{aligned}
-i k_{\rho}\left\langle q\left(\vec{p}_{2}, t_{2}\right) J_{\rho} \bar{q}\left(\vec{p}_{1}, t_{1}\right)\right\rangle= & e^{i k_{4} t_{2}}\left\langle q\left(\vec{q}+\vec{p}_{2}, t_{2}\right) \bar{q}\left(\vec{p}_{1}, t_{1}\right)\right\rangle \delta\left(\vec{k}+\vec{p}_{2}-\vec{p}_{1}\right) \\
& -e^{i k_{4} t_{1}}\left\langle q\left(\vec{p}_{2}, t_{2}\right) \bar{q}\left(\vec{k}-\vec{p}_{1}, t_{1}\right)\right\rangle \delta\left(\vec{p}_{2}-\vec{k}-\vec{p}_{1}\right) .
\end{aligned}
$$

The right hand side is just a linear combination of the incoming and outgoing propagators.

The method just described is slightly different from the original proposal in [5] which required two independent QED ensembles. We calculate our observables using a single QED ensemble to make the two terms in Eq. (2.1) as correlated as possible, and the jack-knife procedure is used to calculate the error on the difference.

In order to calculate the anomalous magnetic moment from the correlation functions described above, we form linear combinations of the electric and magnetic form factors computed at non-zero momentum transfer (for example, see [7]). The external legs are amputated using the two-point functions computed for each source. $F_{2}\left(q^{2}\right)$ needs to be extrapolated to $q^{2}=0$, which has not yet been done in the following.

Again, it is worth emphasizing that the extension to (QED + QCD) is straightforward once the pure QED calculation is shown to work. The calculation goes through in an identical fashion, except that the quark propagators in the loop are computed on a combined QED+QCD configuration. Since the QCD part is identical between the two terms in Eq. (2.1), the added fluctuations should not spoil the delicate cancellation achieved in the pure QED case. 


\section{Preliminary Results}

Our calculations use both Coulomb and Feynman, gauge fixed, non-compact $U(1)$ ensembles and DWF. We averaged over both positive and negative electric charges on the muon line to cancel $\mathscr{O}(e)$ noise configuration by configuration. Our investigations have mostly been limited to $16^{3} \times$ $32 \times 8$ lattices, primarily to search for a signal for $F_{2}$. Once the statistics are under control, we will turn our attention to systematic effects like plateaus and finite size effects. Finally, the calculation was performed for unphysical charge, $e=1$, to enhance the signal, and a rather heavy muon mass of 0.4 . The final result can simply be rescaled by $e^{6}$.

The lepton loop is constructed by inserting the external electromagnetic current at three different time slices, $\left(t_{\mathrm{op}}=4,6,8\right)$. The incoming muon propagator with zero momentum and outgoing propagator with one unit of momentum were created at time slices $t=0$ and 12 , respectively. It was important to check the WTI's before proceeding to calculate the anomalous magnetic moment. They were verified for each on a single non-trivial configuration and in the free case.

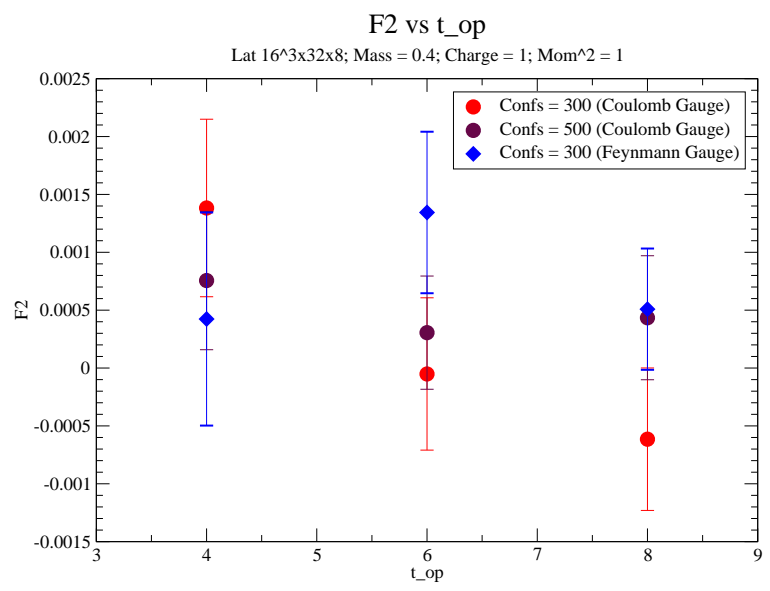

Figure 3: Anomalous magnetic moment $\left(F_{2}\right)$ of muon as a function of time slices of the external vertex $\left(t_{o p}\right)$

Preliminary results are given in Fig. 3 which shows the anomalous magnetic moment, $F_{2}$, as a function of the time slice of the external vertex $t_{o p}$. Red and maroon filled circles are results from Coulomb gauge fixed lattices, and the blue filled diamonds are the Feynman gauged-fixed one. We have done the analysis on 300 and 500 configurations for Coulomb gauge fixed lattices, but only on 300 lattices for the Feynman gauge ${ }^{1}$. It is clear from the figure that more statistics are needed to obtain a signal.

\begin{tabular}{cccc}
\hline & $t_{\mathrm{op}}=4$ & $t_{\mathrm{op}}=6$ & $t_{\mathrm{op}}=8$ \\
\hline$+e$ & $0.0008(9)$ & $-0.00007(82)$ & $0.0004(8)$ \\
$-e$ & $0.0007(9)$ & $0.0007(8)$ & $0.0005(8)$ \\
avg & $0.0008(6)$ & $0.0003(5)$ & $0.0004(5)$ \\
\hline
\end{tabular}

Table 1: Preliminary Results

\footnotetext{
${ }^{1}$ In principle, these two theories of QED may differ when computed in finite volume. [8].
} 
Table 1 shows the results for both positive and negative charges (on the muon line) and the average. The results are insensitive to the charge, so it appears that $\mathscr{O}(e)$ noise is not significant, at least after subtraction. Averaging over the three different values of $t_{o p} \mathrm{~s}$, we find the anomalous magnetic moment from this QED simulation to be $F_{2}=0.00048 \pm 0.00036 . F_{2}$ in perturbation theory has been calculated to be in the order of $\alpha^{3} / \pi^{3}$, which translates into $1.6 \times 10^{-5}$ (for $\mathrm{e}=$ 1). So, the error is one magnitude greater than the perturbative result.

We note in passing that we are here basically simulating the magnetic dipole moment of the electron since the masses of the loop and line were the same. In perturbation theory the magnetic moment only depends on the ratio of masses. Once a robust signal is obtained in this simpler case, we will give unequal masses to the particles in the loop and line.

\section{Conclusion and Acknowledgements}

We have developed the machinery necessary to calculate the light-by-light term on the lattice. Though we did not obtain a signal, we hope to, by increasing our statistics. This can be done in several ways. The simplest way is to increase the number of measurements. However, to reduce the error by the required order of magnitude, or more, would probably be too costly (though more measurements will not hurt). Another approach is to increase the effects of volume averaging by implementing a momentum source for the external source in the loop instead of the point source used here. This requires using noisy sources and/or eigen-modes of the Dirac operator. Finally we can make the volume bigger by moving to larger lattices which will also reduce finite size systematics. All of these methods are now being investigated.

We thank the US Department of Energy and RIKEN for the support necessary to carry out this research. SC and TB were supported by US DOE grant DE-FG02-92ER40716. Computations were carried out on the QCDOC supercomputers at the RIKEN BNL Research Center and Columbia University.

\section{References}

[1] G. W. Bennett [Muon Collaboration], "Final report of the muon E821 anomalous magnetic moment measurement at BNL," Phys. Rev. D 73 (2006) 072003 [arXiv:hep-ex/0602035].

[2] M. Davier and W. J. Marciano, "The theoretical prediction for the muon anomalous magnetic moment," Ann. Rev. Nucl. Part. Sci. 54 (2004) 115.

[3] M. Davier, S. Eidelman, A. Hocker and Z. Zhang, "Updated estimate of the muon magnetic moment using revised results from e+ e- annihilation,” Eur. Phys. J. C 31 (2003) 503 [arXiv:hep-ph/0308213].

[4] K. Melnikov and A. Vainshtein, "Hadronic light-by-light scattering contribution to the muon anomalous magnetic moment revisited," Phys. Rev. D 70 (2004) 113006 [arXiv:hep-ph/0312226].

[5] M. Hayakawa, T. Blum, T. Izubuchi, N. Yamada, "Hadronic light-by-light scattering contribution to the muon g-2 from lattice QCD : Methodology," PoS LAT2005 (2005) 353

[arXiv:hep-lat/0509016v2].

[6] See the talk by P. Rackow at this meeting.

[7] F. Berruto, T. Blum, K. Orginos and A. Soni, Phys. Rev. D 73, 054509 (2006)

[8] M. Hayakawa and S. Uno, arXiv:0804.2044 [hep-ph]. 